\title{
CP Violation at Belle
}

\section{Stephen Schrenk*}

400 Geology/Physics

Cincinnati, $O H$ 45221-0011, USA

E-mail: 'schrenk@phsyics.uc. edu'

Abstract: The Belle collaboration has observed a large signal for $C P$ violation in $B$ meson decays. We used a data set consisting of $29.1 \mathrm{fb}^{-1}$ recorded at the $\Upsilon(4 S)$ resonance collected at the KEKB asymmetric $e^{+} e^{-}$collider. The data set includes 31.3 million $B \bar{B}$ pairs. The mixing-induced $C P$ violation is measured by reconstructing $C P$ eigenstates $\left(J / \psi K_{S}, \psi(2 S) K_{S}, \chi_{c 1} K_{S}, \eta_{c} K_{S}, J / \psi K_{L}\right.$, and $\left.J / \psi K^{* 0}\left(K_{S} \pi^{0}\right)\right)$ from the decay of one $B$ meson while tagging the flavor of the accompanying $B$ meson. From a study of the time-dependent assymetry we find $\sin 2 \phi_{1}=0.99 \pm 0.14 \pm 0.06$, where the first error is statistical and the second systematical.

\section{Introduction}

$C P$ violation was first observed in 1964 in the neutral $K$ meson system by Christenson, Cronin, Fitch and Turlay[i] $\left[\begin{array}{l}{[1} \\ {[}\end{array}\right]$. In 1973, Kobayashi and Maskawa proposed a mechanism for $C P$ violation that required at least three families of quarks [2]. In their mechanism a three by three matrix links the quark mass eigenstates to the weak eigenstates. The matrix elements in this Unitary matrix can be complex. With a three by three matrix one complex phase can not be rotated away allowing the possibility of $C P$ violation.

From the Unitarity requirement of the CKM matrix linking the weak and mass eigenstates one can write down an equation that relates six of the matrix elements: $V_{u d} V_{u b}^{*}+$ $V_{c d} V_{c b}^{*}+V_{t d} V_{t b}^{*}=0$. This relationship can be depicted in the complex plane as a triangle. The area of the triangle is proportional to the amount of $C P$ violation [3.

In 1980 and 1981 Sanda, Carter and Bigi proposed a method for searching for $C P$ violation in the $B$-meson system [4]- Their method utilizes the time dependent mixing of $B^{0}$ and $\bar{B}^{0}$ mesons, producing a time dependent asymmetry in the decay rates of the two $B$ flavors. While elegant in nature, it has taken an additional 20 years to obtain the number of $B$ mesons necessary to confirm $C P$ violation in the $B$ system.

\footnotetext{
${ }^{*}$ Speaker.
} 
We utilize the method of Sanda, Carter and Bigi to measure $C P$ asymmetry. Our data set consists of $29.1 \mathrm{fb}^{-1}$ containing $31.3 \times 10^{6} B \bar{B}$ events collected with the Belle detector located at the KEKB $e^{+} e^{-}$collider. The energy of the collider is tuned to the $\Upsilon(4 S)$ resonance $(\sqrt{s}=10.58 \mathrm{GeV})$. At this resonance two $B$ mesons are produced in a coherent $p$-wave state. When one $B$ decays to a known flavor eigenstate $f_{\text {tag }}, B^{0}$ or $\bar{B}^{0}$, at time $t_{\text {tag }}$ the other $B$ is projected onto the opposite $b$ flavor eigenstate.

The method predicts a decay asymmetry when the other $B$ decays to to a $C P$ eigenstate $f_{C P}$ common to both $B^{0}$ and $\bar{B}^{0}$. When $f_{C P}$ is $(c \bar{c}) K^{(*) 0}$ the expected asymmetry is

$$
A(\Delta t)=\frac{\Gamma\left(\bar{B}^{0} \rightarrow f_{C P}\right)-\Gamma\left(B^{0} \rightarrow f_{C P}\right)}{\Gamma\left(\bar{B}^{0} \rightarrow f_{C P}\right)+\Gamma\left(B^{0} \rightarrow f_{C P}\right)}=-\xi_{f} \sin 2 \phi_{1} \sin \Delta m_{d} \Delta t,
$$

where $\Gamma\left(\bar{B}^{0} \rightarrow f_{C P}\right)$ is the decay rate for $\bar{B}^{0}$ to the common $C P$ eigenstate $f_{C P}$ at a proper time $\Delta t$ defined relative to $t_{t a g}, \xi_{f}$ is the $C P$ eigenvalue of $f_{C P}, \Delta m_{d}$ is the mass difference between the $B^{0}$ meson mass eigenstates, and $\phi_{1}$ is one of the three angles of the Unitarity Triangle defined as $\phi_{1}=\pi-\arg \frac{-V_{t b}^{*} V_{t d}}{-V_{c b}^{*} V_{c d}}\left[\underline{\underline{p}_{1}}\right]$. Decays of the form $(c \bar{c}) K^{(*) 0}$ are clean to reconstruct experimentally and are expected to be easy to interpret theoretically with negligible contributions from direct $C P$ violation and complications from strong interactions.

The time integrated asymmetry is zero requiring us to perform a time dependent measurement. This is done by giving the $\Upsilon(4 S)$ a Lorentz boost in the laboratory rest frame by using asymmetric energy beams $\left(\mathrm{E}_{e^{-}}=8 \mathrm{GeV}, \mathrm{E}_{e^{+}}=3.5 \mathrm{GeV}\right)$. The boost with $\beta \gamma=0.425$ is in the $z$ direction, defined to be along the electron beam line. We can then measure the time between the decays of the two $B$ mesons by measuring the spacial displacement between the two vertices, $\Delta t \simeq\left(z_{C P}-z_{\text {tag }}\right) / \beta \gamma c \equiv \Delta z / \beta \gamma c$.

The KEK B-factory, KEKB, has performed remarkably well. As of the conference date, peak luminosity reached $4.49 \times 10^{33} \mathrm{~cm}^{-2} \mathrm{~s}^{-1}$ (as of the time of writing this proceeding it has reached $\left.5.5 \times 10^{33} \mathrm{~cm}^{-2} \mathrm{~s}^{-1}\right)$. In addition to the $29.1 \mathrm{fb}^{-1}$ of data taken at the $\Upsilon(4 S)$ resonance an additional $3.0 \mathrm{fb}^{-1}$ of data has been taken $60 \mathrm{MeV}$ below the $\Upsilon(4 S)$ resonance to study continuum backgrounds.

The necessary steps for making this measurement are: reconstruct $B^{0} \rightarrow f_{C P}$ candidates, tag the flavor of the other $B\left(f_{\text {tag }}\right)$, measure the vertex displacement between the two $B$ 's and thus the time between their decays, then perform a fit to extract $\sin 2 \phi_{1}$.

\section{Event Selection}

Events are reconstructed with the Belle detector which is described in detail elsewhere [6i The detector consists, from the center outward, of a three layer silicon vertex detector (SVD), a 50 layer central drift chamber (CDC), a mosaic of aerogel Cherenkov detectors for hadron identification (ACC), time-of-flight system (TOF) and a $\mathrm{CsI}(\mathrm{Tl})$ electromagnetic calorimeter (ECL). These are all inside a 1.5 Tesla superconducting coil. The iron flux return is instrumented to detect $K_{L}$ showers and muons (KLM).

Electron identification is based on a combination of CDC $d E / d x$ information, ACC response and the associated ECL shower energy and shape. Muon identification is based on the depth a particle penetrates the KLM and the scatter of KLM hits along the track. 
Kaon and pions are identified by combining CDC $d E / d x$, TOF measurements and ACC response. Photons are identified by ECL showers not matched to a charged track and with a minimum energy of $20 \mathrm{MeV}$.

Events are first selected with a set of criteria designed to elimate non-physics backgrounds. These include requiring at least three "good" charged tracks that come from the interaction point, more than one "good" calorimenter cluster (energy greater than 100 $\mathrm{MeV}$ ), and a balanced momentum in the center of mass. To supress continuum we require $R_{2} \equiv H_{2} / H_{0} \leq 0.5$, where $H_{2}$ and $H_{0}$ are the second and zeroth Fox-Wolfram moments [i]

We reconstruct $B$ mesons in the following $C P$ modes: $J / \psi K_{S}, \psi(2 S) K_{S}, \chi_{c 1} K_{S}$, and $\eta_{c} K_{S}$ for $\xi=-1$ and $J / \psi K_{L}$ for $\xi=1$. We also use $J / \psi K^{* 0}\left(K_{S} \pi^{0}\right)$ which is a mixture of $C P$ even and $C P$ odd final states. The $C P$ content is determined by a full angular analysis of $B \rightarrow J / \psi K^{*}$ decays that excluded the $K^{* 0}$ mode. We measure the $C P \xi=-1$ fraction to be $0.19 \pm 0.04$ (stat) \pm 0.04 (syst) [8i

We reconstruct $J / \psi$ mesons in the $e^{+} e^{-}$and $\mu^{+} \mu^{-}$channels. One lepton is required to be positively identified as an electron or muon, with the other lepton passing a lepton consistency requirement (for electrons a track that satisfies either the electron $d E / d x$ or ECL shower energy requirements, for muons the ECL energy deposit should be consistent with a minimum ionizing particle). Electrons suffer from both final state radiation and bremsstrahlung. In order to reduce the effect of these energy losses we include every photon detected within 0.05 radians from the initial electron direction in the invariant mass calculations. We reconstruct $\psi(2 S)$ in the same way. We defined $J / \psi$ and $\psi(2 S)$ candidates as lepton pairs with an invariant mass between -150 and $+36 \mathrm{MeV} / \mathrm{c}^{2}$ for dielectron and -60 and $+36 \mathrm{MeV} / \mathrm{c}^{2}$ for dimuon candidates of the $J / \psi$ and $\psi(2 S)$ masses. The asymmetric cut is to recover candidates where a radiated photon is missed. The center of mass (com) momentum of $J / \psi$ candidates is required to be less than $2.0 \mathrm{GeV} / \mathrm{c}$, the highest allowed momentum for a $J / \psi$ coming from a $B$-meson decay.

We also reconstruct $\psi(2 S)$ through the $J / \psi \pi^{+} \pi^{-}$channel with the requirement that the $\pi^{+} \pi^{-}$invariant mass be greater than $400 \mathrm{MeV} / \mathrm{c}^{2}$. We then select $\psi(2 S)$ candidates with the mass difference $M_{l^{+} l^{-} \pi^{+} \pi^{-}}-M_{l^{+} l^{-}}$between $0.58 \mathrm{GeV} / 2$ and $0.60 \mathrm{GeV} / 2( \pm 3 \sigma$ in mass resolution). $\chi_{c 1}$ is reconstructed via the $J / \psi \gamma$ channel, where the photons that are consistent with $\pi^{0} \rightarrow \gamma \gamma$ are vetoed. We select $\chi_{c 1}$ candidates with mass difference $M_{l^{+} l^{-} \gamma}-M_{l^{+} l^{-}}$between $0.3850 \mathrm{GeV} /{ }^{2}$ and $0.4305 \mathrm{GeV} /{ }^{2}$. For $\psi^{\prime}$ and $\chi_{c 1}$ both leptons must be positively identified. Histograms of invariant mass spectra can be seen in Fig. İi through Fig. 迎. Note the clear signal for $B \rightarrow \chi_{c 2} X$ in Fig. 岱. This is the first observation of this decay.

The $\eta_{c}$ is reconstructed in the $K^{+} K^{-} \pi^{0}$ and $K_{S} K^{-} \pi^{+}$modes. As the modes are hadronic, tighter selection criteria are used. Charged kaons are required to be positively identified using combined information from CDC $d E / d x$, TOF, and ACC information. For the $K^{+} K^{-} \pi^{0}$ mode we require the invariant mass of the $\eta_{c}$ candidate to be between 2.890 and $3.040 \mathrm{GeV} / \mathrm{c}^{2}$. To suppress background from continuum we require $R_{2}<0.45$ and $\left|\cos \theta_{t h r}\right|<0.85$ where $\theta_{t h r}$ is the thrust axis define by the $B^{0}$ candidate and that of the rest of the event. For the $K_{S} K^{-} \pi^{-}$mode we require the invariant mass to be between 2.935 and $3.035 \mathrm{GeV} / \mathrm{c}^{2}, R_{2}<0.40$, and $\left|\cos \theta_{t h r}\right|<0.85$. 


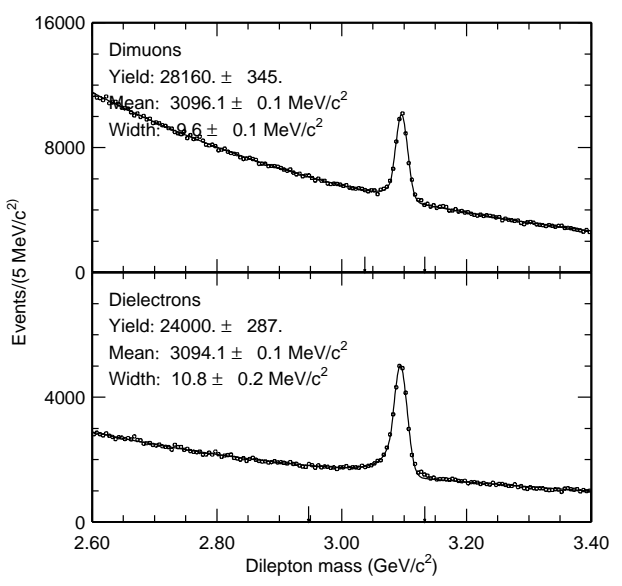

Figure 1: Dilepton invariant mass spectra in $J / \psi$ mass region. One lepton has been positively identified while the other has passed a lepton consistency requirement.

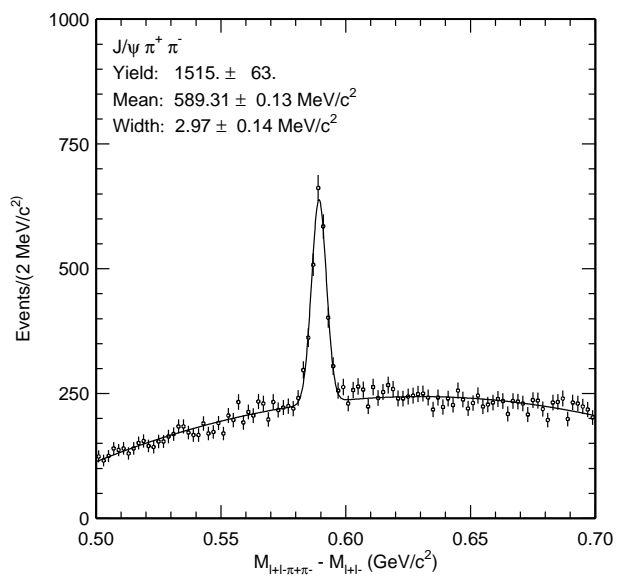

Figure 3: Invariant mass difference between $\psi(2 S)$ candidate and $J / \psi$ candidate.

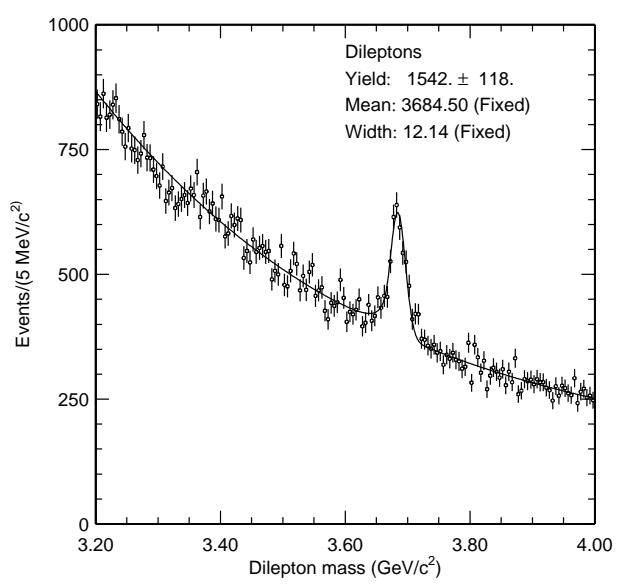

Figure 2: Dilepton invariant mass spectra in $\psi(2 S)$ region. Both leptons have been positively identified.

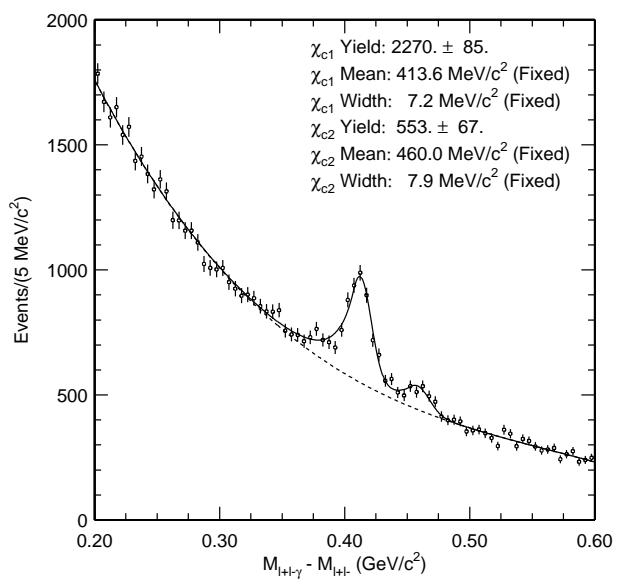

Figure 4: Invariant mass difference between $\chi_{c}$ candidate and $J / \psi$ candidate.

Charmonium candidates are then paired with $K_{S}\left(\pi^{+} \pi^{-}\right)$candidates to form $B$-meson candidates. These $K_{S}$ candidates are identified by a displaced $\pi^{+} \pi^{-}$vertex and a mass within $4 \sigma$ of the $K^{0}$ mass $\left(\sigma \approx 4 \mathrm{MeV} / c^{2}\right)$. In addition, we reconstruct $B$ mesons by combining $J / \psi$ candidates with $K_{S}\left(\pi^{0} \pi^{0}\right), K_{L}$, or $K^{* 0}\left(K_{S} \pi^{0}\right)$ candidates. For $K_{S}\left(\pi^{0} \pi^{0}\right)$, all combinations of two $\gamma \gamma$ pairs with an invariant mass between 80 and $150 \mathrm{MeV} / \mathrm{c}^{2}$ are tried, assuming that the photons originate from the interaction point. We then minimize the sum of $\chi^{2}$ 's coming from fitting the pairs of photons assuming a $\pi^{0}$ mass, varying the decay point along the $K_{S}$ flight path which is assumed to be along a line from the interaction point to the energy-weighted center of the four showers. For $K_{L}$, we define a candidate to be a shower in the ECL and/or KLM that is not matched to a charged track and has a hit pattern consistent with coming from a hadronic shower. For $K^{* 0}$, we define a candidate as a $K_{S}$ and $\pi^{0}$ pair with an invariant mass with in $75 \mathrm{MeV} / \mathrm{c}$ of the $K^{* 0}$ mass. Background from low momentum $\pi^{0}$ 's is reduced by requiring $\cos \theta_{K^{*}}<0.8$, where $\theta_{K^{*}}$ is 
the angle between the $K_{S}$ direction and the $K^{* 0}$ direction calculated in the $K^{* 0}$ rest frame.

We define $B$-meson candidates for all modes except $J / \psi K_{L}$ via two kinematic variables. The first variable is $\Delta \mathrm{E}$, defined as the difference between the energy of the $B$ candidate and the beam energy in the center of mass. $B$ candidates are required to have a $\Delta \mathrm{E}$ consistent with zero. The exact criteria depends on the mode and is typically with \pm 40 $\mathrm{MeV}$ (larger values for modes with $\pi^{0}$ 's). Since at the $\Upsilon(4 S)$ only two $B$ mesons are produced the $B$ energy must match the beam energy. The second variable is the "beam constrained mass" (bc). This is the invariant mass of the $B$ candidate where the beam energy has been used instead of the $B$-candidate energy. This considerably improves the mass resolution. $B$ candidates are required to have a beam constrained mass between 5.270 and $5.280 \mathrm{GeV} / \mathrm{c}^{2}$. A list of the number of candidates for each mode can be found in Table i: Fig.

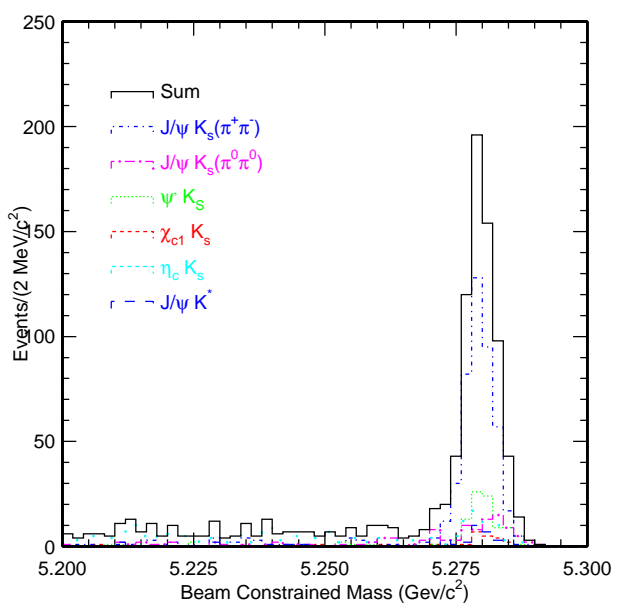

Figure 5: Beam-energy constrained mass plot for fully reconstructed modes (all but $\left.J / \psi K_{L}\right)$.

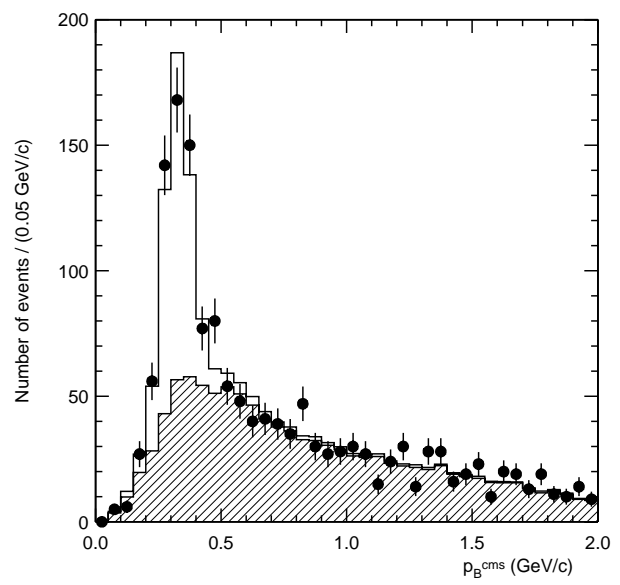

Figure 6: $B$-candidate momentum in center of mass rest frame for $B \rightarrow J / \psi K_{L}$.

Reconstruction of $J / \psi K_{L}$ candidates is a bit more difficult as the $K_{L}$ momentum is not directly measured. We begin by requiring that the centroid of the $K_{L}$ candidate shower be within $45^{\circ}$ of the inferred flight direction from the two body decay $B \rightarrow J / \psi K_{L}$. The background is reduced through a likelihood ratio composed of the $J / \psi$ com momentum, the angle of the $K_{L}$ and its nearest neighbor charged track, the charged track multiplicity of the event, the extent the event is consistent with $B^{+} \rightarrow J / \psi K^{*+}\left(K_{L} \pi^{+}\right)$, and the polar angle of the reconstructed $B$ with respect to the $z$ direction of the reconstructed $B^{0}$ in the com. We also remove events that are reconstructed as $B^{0} \rightarrow K_{S}, J / \psi K^{* 0}\left(K^{+} \pi^{-}, K_{S} \pi^{0}\right)$, $B^{+} \rightarrow J / \psi K^{+}$, or $J / \psi K^{*+}\left(K^{+} \pi^{0}, K_{S} \pi^{+}\right)$decays. $K_{L}$ clusters that match a photon that reconstructs with another photon to form a $\pi^{0}$ candidate are also rejected.

Next we calculate the momentum of the $B$ candidate in the com assuming the $B^{0} \rightarrow$ $J / \psi K_{L}$ hypothesis $\left(p_{B}^{c o m}\right)$. This distribution is shown in Fig. ' $6 \sigma_{\mathbf{r}}^{-1}$ The histogram shows a fit to a combination of signal and background line shapes that were determined by a Monte Carlo simulation where the normalization and peak position were allowed to vary. For $K_{L}$ candidates that contain KLM hits, the signal region is defined to be $0.2 \leq p_{B}^{\text {com }} \leq$ 
$0.45 \mathrm{GeV} / c$. There are 397 candidate events in this region. For $K_{L}$ candidates that contain only ECL hits the signal region is $0.2 \leq p_{B}^{c o m} \leq 0.40 \mathrm{GeV} / c$. There are 172 events in this region. The combined fit give a total of $(346 \pm 29) J / \psi K_{L}$ signal events with a purity of $61 \%$.

\section{Flavor Tagging}

Once we have our $C P B$-candidates we must tag the flavor of the other $B$ meson. The tag exploits the kinematics of the $b \rightarrow$ $c \rightarrow s$ quark decay chain that reflect the flavor of the $b$. We look for high momentum leptons from $b \rightarrow \mathrm{cl}^{-} \bar{\nu}$, low momentum leptons from $c \rightarrow s l^{+} \nu$, charged kaons and $\Lambda$ baryons from $c \rightarrow s X$, high momentum pions from $B \rightarrow D^{(*)} h$ where $h$ is a $\pi^{+}, \rho^{+}, a_{1}^{+}$, etc., and slow pions from $D^{*-} \rightarrow \bar{D}^{0} \pi^{-}$.

For each of these categories tagging methods we use MC to determine a method-

\begin{tabular}{lrr}
\hline \hline Mode & $N_{e v}$ & $N_{\text {bkgd }}$ \\
\hline$J / \psi\left(l^{+} l^{-}\right) K_{S}\left(\pi^{+} \pi^{-}\right)$ & 457 & 11.9 \\
$J / \psi\left(l^{+} l^{-}\right) K_{S}\left(\pi^{0} \pi^{0}\right)$ & 76 & 9.4 \\
$\psi(2 S)\left(l^{+} l^{-}\right) K_{S}\left(\pi^{+} \pi^{-}\right)$ & 39 & 1.2 \\
$\psi(2 S)\left(J / \psi \pi^{+} \pi^{-}\right) K_{S}\left(\pi^{+} \pi^{-}\right)$ & 46 & 2.1 \\
$\chi_{c 1}(J / \psi \gamma) K_{S}\left(\pi^{+} \pi^{-}\right)$ & 24 & 2.4 \\
$\eta_{c}\left(K^{+} K^{-} \pi^{0}\right) K_{S}\left(\pi^{+} \pi^{-}\right)$ & 23 & 11.3 \\
$\eta_{c}\left(K_{S} K^{-} \pi^{+}\right) K_{S}\left(\pi^{+} \pi^{-}\right)$ & 41 & 13.6 \\
$J / \psi\left(l^{+} l^{-}\right) K^{* 0}\left(K_{S} \pi^{0}\right)$ & 41 & 6.7 \\
\hline Subtotal & 747 & 58.6 \\
\hline$J / \psi\left(l^{+} l^{-}\right) K_{L}$ & 569 & 223 \\
\hline \hline
\end{tabular}

Table 1: The number of observed event candidates $\left(N_{e v}\right)$ and the estimated background $\left(N_{b k g d}\right)$ dependent variable that reflects if a track originated from a $B^{0}$ or $\bar{B}^{0}$. The particles charge, com momentum, polar angle and particleidentification probability, as well as event level properties go into determining this variable. The variables range continuously between -1 and +1 with a value of -1 indicating a reliably identified $\bar{B}^{0}$ and a value of +1 indicating a reliably identified $B^{0}$.

The results of the separate methods are then combined together taking into account correlations. The output of this level is a value ' $q$ ' that is -1 if the tag-side $B$ is more $B^{0}$ like or -1 if it is more $\bar{B}^{0}$ like. A second value ' $r$ ' is output giving the quality of the tag, 0 if there is no flavor discrimination and 1 if there is perfect discrimination. The value $r$ is used to divide the data into six groups according to flavor purity.

The incorrect flavor assignment probability $w_{l}$ is determined for each bin of $r$ from data using self-tagged decays $\left(B^{0} \rightarrow D^{*-} l^{+} \nu, D^{(*)-} \pi^{+}\right.$, and $D^{*-} \rho^{+}$. The values of $w_{l}$ are determined by the amplitudes of the time-dependent $B^{0} \bar{B}^{0}$ mixing oscillations described by: $\frac{N_{O F}-N_{S F}}{N_{O F}+N_{S F}}=\left(1-2 w_{l}\right) \cos \left(\Delta m_{d} \Delta t\right)$, where $N_{O F}$ is the number of events with opposite flavor $B$ 's and $N_{S F}$ is the number of events with the same flavor $B$ 's. In performing the fits, $\Delta m_{d}$ is fixed to the world average value [1] $\left.{ }_{1}^{i}\right]$. The total tagging efficiency is then then $\sum_{l} f_{l}\left(1-2 w_{l}\right)^{2}=0.270 \pm 0.008$ (stat) ${ }_{-0.009}^{+0.006}$ (syst) where $f_{l}$ is the fraction of events in each bin of $r$. A summary of $f_{l}$ and $w_{l}$ can be found in Table $\overline{2}$.

\section{Vertexing}

The next step is to find the time difference between the two $B$ decays which requires us to find the vertex of the two $B$ 's. We only use tracks that have both $r-\phi$ and $z$ hits in 
the same SVD layer along with one or more additional $z$ hits in the other layers. Both vertices are required to be consistent with the interaction point profile taking into account the $B$ meson decay length. For the $f_{C P} B$ only the tracks that come from the charmonium candidate are used: lepton tracks for $J / \psi$ and $\psi(2 S)$ and prompt hadron tracks for $\eta_{c}$. The efficiency and the $z$ vertex resolution (rms) are determined from Monte Carlo and found to be $95 \%$ and $75 \mu \mathrm{m}$ (measured with $B^{+} \rightarrow J / \psi K^{+}$and $B^{0} \rightarrow J / \psi K^{* 0}\left(K^{+} \pi^{-}\right)$data events). For the $f_{\text {tag }} B$ we use all tracks not associated with the $f_{C P} B$ and not associated with a $K_{S}$ decays. Tracks that contribute a large $\chi^{2}$ to the vertex fit are eliminated in an iterative process. The efficiency and $z$ vertex resolution for the $f_{C P} B$ are $93 \%$ and 140 $\mu \mathrm{m}$ (also measured with $B^{+} \rightarrow J / \psi K^{+}$and $B^{0} \rightarrow J / \psi K^{*} 0\left(K^{+} \pi^{-}\right)$data events).

Once the two vertices have been found, the proper-time interval is calculated. The resolution of the proper-time interval $R_{\text {sig }}$ is an important input in to the $C P$ fit. It is approximated by the sum of two Gaussians, a main Gaussian that is due to the SVD vertex resolution and charm meson lifetimes, and a tail Gaussian due to poorly reconstructed tracks. The relative fractions of the two Gaussians is determined from a study of $B^{0} \rightarrow D^{*-} \pi^{+}, D^{*-} \rho^{+}, D^{-} \pi^{+}, J / \psi K_{S}$, and $B^{+} \rightarrow \bar{D}^{0} \pi^{+}, J / \psi K^{+}$events. The main component fraction is $0.97 \pm 0.02$. The mean and errors

\begin{tabular}{cccc}
\hline \hline$l$ & $r$ & $f_{l}$ & $w_{l}$ \\
\hline 1 & $0.000-0.250$ & 0.405 & $0.465_{-0.009}^{+0.010}$ \\
2 & $0.250-0.500$ & 0.149 & $0.352_{-0.014}^{+0.015}$ \\
3 & $0.500-0.625$ & 0.081 & $0.243_{-0.021}^{+0.020}$ \\
4 & $0.625-0.750$ & 0.099 & $0.176_{-0.022}^{+0.017}$ \\
5 & $0.750-0.875$ & 0.123 & $0.110_{-0.022}^{+0.014}$ \\
6 & $0.875-1.000$ & 0.140 & $0.041_{-0.010}^{+0.011}$ \\
\hline
\end{tabular}

Table 2: For each bin of ' $r$ ', the event fraction $f_{l}$ and $w_{l}$ are listed. Errors include both statistical and systematic uncertainties.

of the Gaussians are calculated on an event-by-event basis from the vertex fit error matrices and the $\chi^{2}$ of the fit. Typical values for the main (tail) component are $-0.24 \mathrm{ps}(0.18 \mathrm{ps})$ for the mean and $1.49 \mathrm{ps}(3.85 \mathrm{ps})$ for the error.

The "resolution" for background events $R_{b k g}(t)$ is also a necessary input for the $C P$ fit. The shape is also described by a double Gaussian. The parameters are determined by studying the sidebands in $M_{b c}$ and $\Delta E$.

To verify our procedure for finding the vertex resolutions we measure the $B$ meson lifetimes using the same resolutions and techniques. We find $\tau_{B^{0}}=1.547 \pm 0.021$ and $\tau_{B^{+}}=1.641 \pm 0.033 p s$ where the errors are statical only.

\section{Fitting Procedure}

We perform an unbinned likelihood fit to find $\sin 2 \phi_{1}$. The fit includes the vertex resolutions for signal and background, mistagging fraction, $C P$ state of $B$, and for $J / \psi K^{*}$ the transversity angle.

After vertexing we find 560 events with $q=+1$ and 577 events with $q=-1$ flavor tags. A clear asymmetry can be seen in the raw data when distributions in $\Delta t$ are made for $q \xi_{f}=+1$ and $q \xi_{f}=-1$ separately as seen in Fig. $\underline{\underline{7}}$. This in itself shows that $C P$ symmetry is violated. 
In order to determine $\sin 2 \phi_{1}$ we perform an unbinned maximum likelihood fit. The probability density function (PDF) for signal events for all modes except $B \rightarrow J / \psi K^{* 0}$ is

$$
\mathcal{P}_{\text {signal }}\left(\Delta t, q, w_{l}, \xi_{f}\right)=\frac{e^{-|\Delta t| / \tau_{B^{0}}}}{2 \tau_{B^{0}}}\left\{1-\xi_{f} q\left(1-w_{l}\right) \times \sin 2 \phi_{1} \sin \left(\Delta m_{d} \Delta t\right)\right\} .
$$

For $J / \psi K^{* 0}$ we include the $\Delta t$ and transversity angle $\left.\theta_{t r}[\underline{\underline{9}}]\right]$ in the likelihood and use the $\xi_{f}$ content from the full angular analysis. The values of $\Delta m_{d}$ and $\tau_{B^{0}}$ are fixed to the world averages, $\Delta m_{d}=0.472 \mathrm{ps}^{-1}$ and $\tau_{B^{0}}=1.55 \mathrm{ps}[\underline{1} \underline{1} \underline{0} \overline{0}$.

For background we use

$$
\mathcal{P}_{b k g}(\Delta t)=f_{\tau} \frac{e^{-|\Delta t| / \tau_{b k g}}}{2 \tau_{b k g}}+\left(1-f_{\tau}\right) \delta(\Delta t),
$$

where $f_{\tau}$ is the fraction of the background with a non-zero effective lifetime $\tau_{b k g}$ and $\delta$ is the Dirac delta function. Looking at the regions in $M_{b c}$ and $\Delta E$ where background dominates we find that $f_{\tau}$ is effectively zero for all modes but $J / \psi K_{L}$.

For $J / \psi K_{L}$, the backgrounds are dominated by inclusive $B \rightarrow J / \psi X$ decays. We use Monte Carlo to determine the make up of this background and the relative amounts of the various modes that are $C P$ eigenstates. We find $71 \%$ of the background is non- $C P$ modes with $\tau_{b k g}=\tau_{B^{0}}$. Another $13 \%$ of the background is $J / \psi K^{* 0}\left(K_{L} \pi^{0}\right)$. For this component we use the $\xi_{f}$ content determined by the full angular analysis of $B \rightarrow J / \psi K^{*}$. Events with $\xi_{f}=+1$ make up $5 \%$ of the background while events with $\xi_{f}=-1$ make up $10 \%$.

The final step is to maximize the likelihood

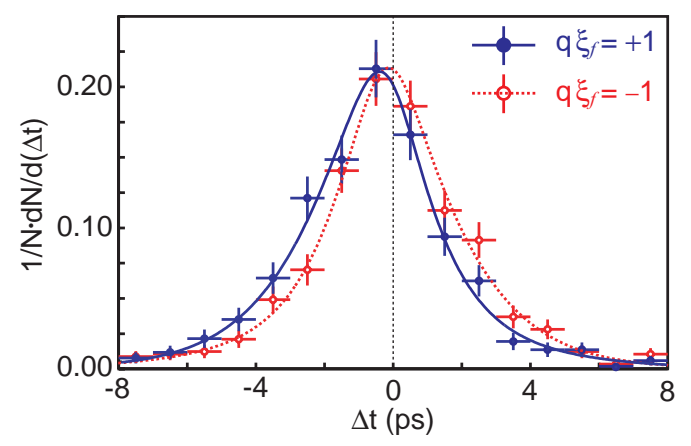

Figure 7: The $\Delta t$ distribution for events with $q \xi_{f}=+1$ (red solid points) and $q \xi_{f}=$ -1 (blue open points). The distribution is overlayed with the results of the global fit. $L=\Pi_{i} P_{i}$ where

$$
P_{i}=\int\left\{f_{\text {sig }} \mathcal{P}_{\text {sig }}\left(\Delta t^{\prime}, q, w_{l}, \xi_{f}\right) R_{\text {sig }}\left(\Delta t-\Delta t^{\prime}\right)+\left(1-f_{\text {sig }}\right) \mathcal{P}_{\text {bkg }}\left(\Delta t^{\prime}\right) R_{b k g}\left(\Delta t-\Delta t^{\prime}\right)\right\} d \Delta t^{\prime},
$$

where $f_{\text {sig }}$ is the probability that an event is signal as determined by $M_{b c}$ and $\Delta E$ for all decays except $J / \psi K_{L}$ where $p_{B}^{\text {com }}$ is used. The only free parameter in the fit is $\sin 2 \phi_{1}$. We find

$$
\sin 2 \phi_{1}=0.99 \pm 0.14(\text { stat }) \pm 0.06 \text { (syst) }
$$

It is perhaps easiest to demonstrate the significance of this fit by overlaying the curve derived from this fit on top of asymmetries determined for individual bins of $\Delta t$. This is

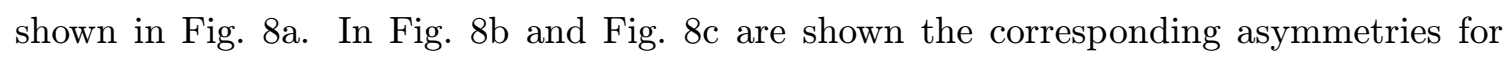
events with $\xi_{f}=-1$ (all events except $J / \psi K_{L}$ and $J / \psi K^{* 0}$ ) and for events with $\xi_{f}=+1$ $\left(J / \psi K_{L}\right)$. The values of $\sin 2 \phi_{1}$ determined for each of these subsets of events are $0.84 \pm 0.17$ and $1.31 \pm 0.23$ respectively, where the errors are statistical only. 


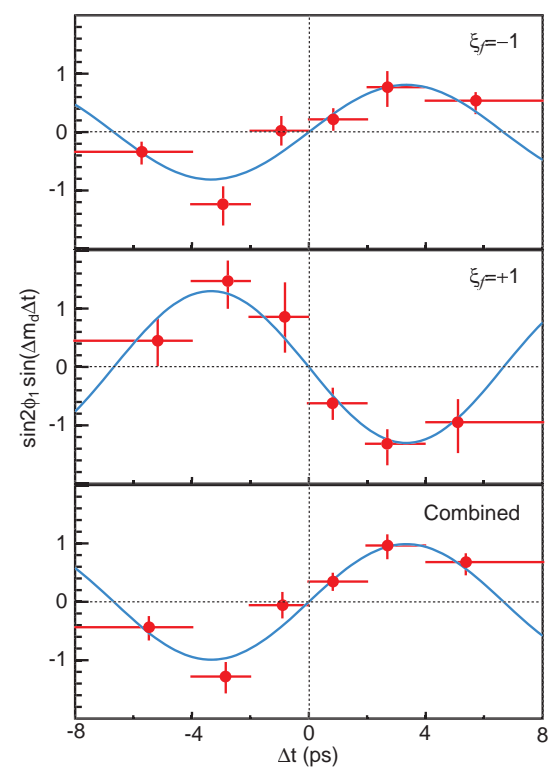

Figure 8: Time dependent asymmetry.

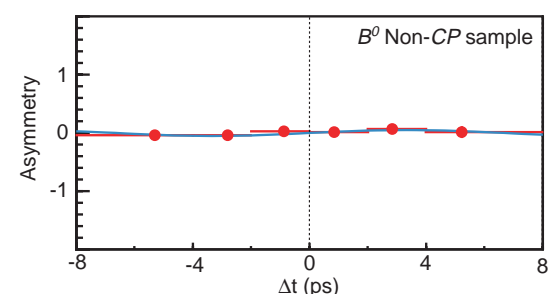

Figure 9: Time dependent asymmetry for a control sample.

A variety of check have been performed for this measurement. An obvious check is to perform the fit to a set of non- $C P$ events that are self tagged where the expected asymmetry is zero. When we fit to a set of such events that include $B^{0} \rightarrow D^{(*)-} \pi^{+}$, $D^{*-} \rho^{+}, J / \psi K^{* 0}\left(K^{+} \pi^{-}\right)$, and $D^{*-} l^{+} \nu$, we find a value of $0.05 \pm 0.04$. The asymmetry for this control sample is shown in Fig. '的; Other checks involve breaking the event sample into different components. The results of these fits are shown in Table

\begin{tabular}{lrrrr}
\hline \hline Sample & $\sin 2 \phi_{1}$ & & Source & Contribution \\
\cline { 1 - 2 } \cline { 1 - 2 }$f_{\text {tag }}=B^{0}(q=+1)$ & $0.84 \pm 0.21$ & & Vertex Algorithm & \pm 0.04 \\
$f_{\text {tag }}=\bar{B}^{0}(q=-1)$ & $1.11 \pm 0.17$ & & Flavor Tagging & \pm 0.03 \\
$J / \psi K_{S}\left(\pi^{+} \pi^{-}\right)$ & $0.81 \pm 0.20$ & & Resolution Function & \pm 0.02 \\
$c \bar{c} K_{S}$ except $J / \psi K_{S}\left(\pi^{+} \pi^{-}\right)$ & $1.00 \pm 0.40$ & & Background Fraction $K_{L}$ & \pm 0.02 \\
$J / \psi K_{L}$ & $1.31 \pm 0.23$ & & Background Shape & \pm 0.01 \\
$J / \psi K^{* 0}\left(K_{S} \pi^{0}\right)$ & $0.85 \pm 1.45$ & & $\Delta m_{d}$ and $\tau_{B^{0}}$ errors & \pm 0.01 \\
\hline All & $0.99 \pm 0.14$ & & Total & \pm 0.06 \\
\hline \hline
\end{tabular}

Table 3: The values of $\sin 2 \phi_{1}$ for various subsamples. The errors are statistical only.

Table 4: Sources of Systematic Errors.

We have also performed checks where the values of $\Delta m_{d}$ and $\tau_{B^{0}}$ are allowed to float. We find for the former case a value of $\sin 2 \phi_{1}$ of $1.00 \pm 0.14$ with a value of $\Delta m_{d}$ of $0.478 \pm 0.057$. For the latter case we find $\sin 2 \phi_{1}=1.00 \pm 0.14$ with a value of $\tau_{B^{0}}$ of $1.66 \pm 0.07 \mathrm{ps}$. We also allowed for direct $C P$ violation. We find the fit result consistent with no direct $C P$ violation and the value of $\sin 2 \phi_{1}$ to be unchanged. 


\section{Summary}

We have measured a large $C P$ violation in the neutral $B$-meson system. The value is consistent with the largest value of $C P$ violation allowed in the $\mathrm{KM}$ model. A value of zero is ruled out at a statistical significance of more than $6 \sigma$. In the process of performing this measurement we have also observed for the first time $B \rightarrow \chi_{c 2} X$ production.

A comparison of our measurement with measurements of $\sin 2 \phi_{1}$ from other experiments, as well as a combined value can be seen in Fig. violation occurs in the $B$-meson system as well as in the $K$-meson system. Kobayashi and Maskawa's model has been a stunning success in describing basic quark interac-

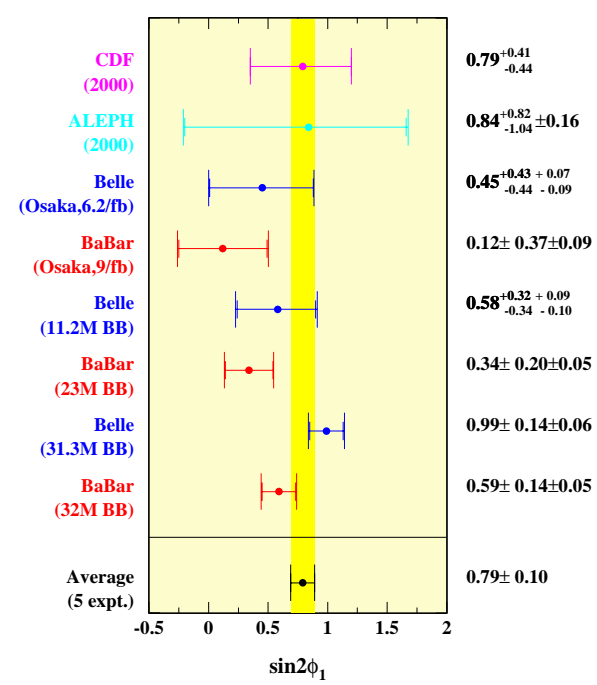

Figure 10: Comparison with other experiments. tions.

\section{References}

[1] J. H. Christenson, J. W. Cronin, V. L. Fitch and R. Turlay, 'Phys. Rev. Lett. 13. 1964 4 138

[2] M. Kobayashi and T. Maskawa, 'Prog. Theor.

[3] C. Jarlskog, 'Phys. Rev. Lett. $55 \overline{5}(1985)$ 1039!

[4] A. B. Carter and A. I. Sanda, P'phys.Rev. Lett. $45 \overline{5}(19 \overline{9} 0) \overline{9} 52$; A. B. Carter and A. I. Sanda, 'Phys. Rev. D 23 1981$) 156 \%$ I. I. Bigi and A. I. Sanda, Nucl. Phys. B 193

[5] H. Quinn and A. I. Sanda, 'Eur. Phys. J. C $\mathbf{1} 5(2000) 626$.

[6] K. Abe et al. (Belle Collaboration), KEK Report 2000-4, to be published in Nucl. Instrum. Methods Phys. Res.

[7] G. C. Fox and S. Wolfram, iPhys. Rev. Lett. 41 (1978) $\overline{1} \overline{8} \overline{1}$.

[8] K. Abe et al. (Belle collaboration), BELLE-CONF-0015 (2001).

[9] I. Dunietz, H. R. Quinn, A. Snyder, W. Toki, and H. J. Lipkin, Phys. Rev.

[10] D.E. Groom et al., The European Physical Journal C15 (2000) 1, and 2001 off-year partial update for the 2002 edition available on the PDG WWW pages (URL: http://pdg.lbl.gov/).

[11] K. Ackerstaff et al (OPAL collaboration), 'Eur.Phys. J. 5 (1998) 379 ; T. Affolder et al (CDF collaboration), 'P $\bar{P} y s$. Rev. $\mathbf{D} \mathbf{6} \overline{\mathbf{1}}(2000) 07200 \overline{5} ; \mathrm{B}$. Barate et al (ALEPH

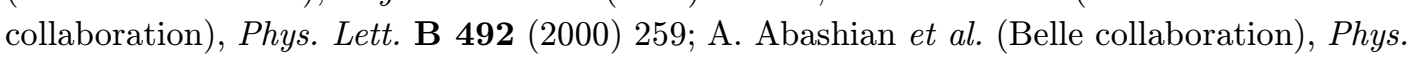

Rev. Lett. 86 (2001)2509; B. Aubert et al. (BaBar collaboration), P'Phys. Rev. Lett. 86. 2001)

2 - 2515i; B. Aubert et al. (BaBar collaboration), 'Phys. Rev. Lett. 87. (2001) 091801' K. Abe et al. (Belle collaboration), 'Phys. Rev. Lett. 87. (2001) 091802. 